\title{
Le vignoble du Mâconnais, entre Côte d'Or et Beaujolais, approche géographique
}

Mâconnais's vineyard between Côte d'Or and Beaujolais. Geographical study Der Weinbau im Mâconnais zwischen Côte d'Or und Beaujolais, ein geographischer Essay

Gérard Mottet

\section{(2) OpenEdition}

\section{Journals}

Édition électronique

URL : http://journals.openedition.org/rge/1003

DOI : $10.4000 /$ rge. 1003

ISSN : 2108-6478

Éditeur

Association des géographes de l'Est

Édition imprimée

Date de publication : 1 janvier 2004

Pagination : 31-42

ISSN : 0035-3213

\section{Référence électronique}

Gérard Mottet, «Le vignoble du Mâconnais, entre Côte d'Or et Beaujolais, approche géographique », Revue Géographique de l'Est [En ligne], vol. 44 / 1-2 | 2004, mis en ligne le 10 juin 2009, consulté le 10 décembre 2020. URL : http://journals.openedition.org/rge/1003 ; DOI : https://doi.org/10.4000/rge. 1003

Ce document a été généré automatiquement le 10 décembre 2020.

Tous droits réservés 


\section{Le vignoble du Mâconnais, entre Côte d'Or et Beaujolais, approche géographique}

Mâconnais's vineyard between Côte d'Or and Beaujolais. Geographical study

Der Weinbau im Mâconnais zwischen Côte d'Or und Beaujolais, ein

geographischer Essay

\section{Gérard Mottet}

\section{NOTE DE L'ÉDITEUR}

Article reçu le 16 mai 2004 ; accepté le 12 juin 2004

L'auteur remercie particulièrement Cédric Fermond, ingénieur au centre INAO de Mâcon qui a réalisé pour la commission d'experts le " rapport général sur l'AOC Mâcon 2004 », rapport auquel cet article doit beaucoup.

\section{Introduction}

1 Entre les prestigieux " premiers crus » et " grands crus » de la « Côte bourguignonne » et le plus populaire et médiatique Beaujolais au Sud, le vignoble du Mâconnais et ses nombreuses appellations communales, tiennent, dans l'espace géographique viticole bourguignon, une place très particulière.

2 L'histoire, la géographie physique et la hiérarchisation complexe de la procédure des appellations d'origine contrôlée (AOC) sont à l'origine de cette spécificité. 


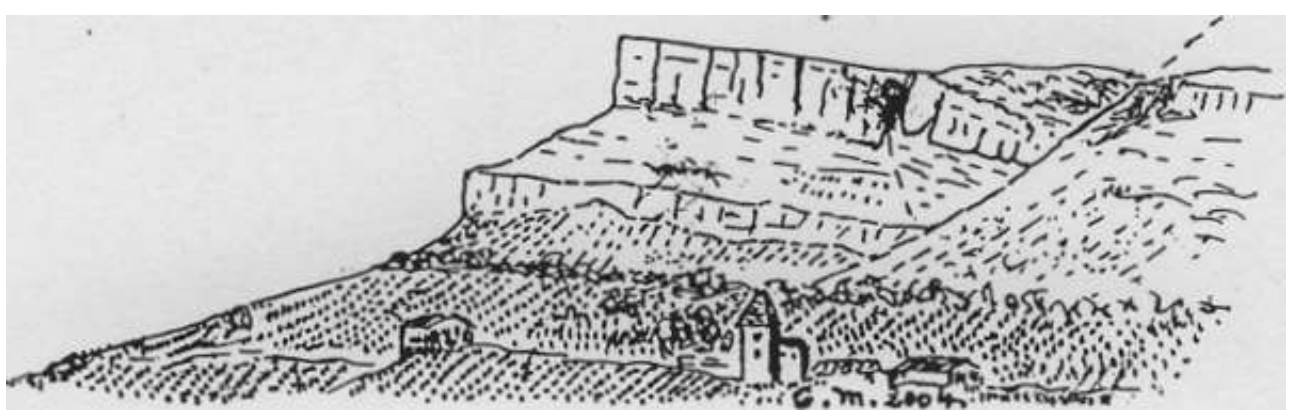

\section{Données historiques et contraintes fiscales d'Ancien Régime}

3 Avant d'être un axe essentiel de la conquête romaine, le couloir Saône-Loire l'a été de la rencontre des peuples celtes et de la civilisation grecque, première responsable de la remontée de la vigne dans le monde forestier de l'Europe atlantique. Les gaulois y ajoutèrent l'art de la tonnellerie plus propice au transport du vin que les amphores $d u$ monde gréco-romain.

Bien situé sur le golfe de remontée des influences climatiques méditerranéennes, le Mâconnais a pu très tôt, à l'ouest de la Saône, sur ses collines calcaires, accueillir Vitis Vinifera dans sa forme originelle d'Asie occidentale et d'Europe méditerranéenne; notamment par la présence grecque en Provence sous sa forme la plus diffusée dès le V ${ }^{\mathrm{e}}$ siècle avant J.C., Vitis vinifera caucasica.

Comme tous les vignobles du couloir rhodanien et saônois, le vignoble du Mâconnais a ensuite été associé à la romanisation de la Gaule, même si, de nos jours, d'anciens cépages que la tradition affirme être venus de la Méditerranée romanisée (césar, syrah), ne sont présents qu'à titre de témoignage historique en Bourgogne. Le Mâconnais était fort bien situé sur cet axe stratégique qui, de Lugdunum (Lyon) à Colonia Augusta (Cologne), a constitué une voie historique structurante essentielle de la conquête romaine et de ses conséquences économiques. Bien des vignobles de l'actuelle France de l'Est, de la vallée de la Moselle et de l'axe rhénan doivent certainement leur origine à ces voies romaines qui, parties de la capitale des Gaules, gagnaient le Rhin, par Matisco (Mâcon) Dibio (Dijon) Grannos (Grand), Civitas Lencorum (Toul), Augusta Treverorum (Trèves). Il en fut de même pour la vallée du Rhin moyen et l'Alsace à partir de la " Porte de Bourgogne ", la porta occidentalis des anciens (Georges CHABOT, la Bourgogne, Armand Colin, 1941, p. 217).

Incontestablement tous les travaux d'archéologie, de préhistoire et d'histoire ancienne tendent à confirmer ce rôle essentiel de liant, "d'axe de civilisation» de la Saône depuis la plus haute antiquité et du commerce fluvial qui y était lié, et notamment du vin, qu'il fût d'origine méditerrannéenne puis locale, c'est-à-dire gauloise, puis "bourguignonne " et "mâconnaise ", lorsque ces mêmes territoires seront consacrés par la toponymie et l'histoire médiévales (1). André Pelletier rappelle dans ce volume (pp. 329-333) l'importance des "nautes de la Saône » et des cinq corporations qui se partageaient le commerce sur la rivière. Certains de ces nautes ou bateliers, à l'exemple de Caius Sentius Regulus, sont des négociants en vins, et Caius Apronius Raptor est à ce titre en plus patron de la Corporation. Dans le même travail, A. Pelletier (La Saône, axe 
de civilisation, PUL 552 p. nov. 2002) rappelle également que ces nautes transportent déjà au profit du marché de Lugdunum «le vin qui provient de Bourgogne où le vignoble existe depuis le Ier siècle (découverte d'un atelier d'amphores vinaires à Gueugnon) ». Il est vrai que de nombreuses assises argileuses tapissent le sillon houiller Dheune-Bourbince débouchant sur le val de Loire à l'ouest et le val de Saône à l'est.

Ce goût pour le vin et son commerce dans l'axe de la Saône, dès la plus haute Antiquité, est également confirmé par la fréquence des restes d'amphores vinaires dans les vestiges de l'oppidum de Matisco, d'origine méditerranéenne, mais aussi de production gauloise (A. Barthélémy, 1967, 1968-69 et 1973).

La culture de la vigne et le commerce du vin se continuèrent pendant tout le MoyenAge, affaibli pendant les grandes invasions, développés par l'Église, les grands ordres monastiques et notamment par l'Ordre de Cluny si proche du Mâconnais (Marcel Pacaut, l'Ordre de Cluny, 1994, p. 273) : la production viticole, au X ${ }^{\mathrm{e}}$ siècle, à l'aube de la puissance clunysienne, atteint « 380 muids en moyenne » dans le vignoble d'alors où l'on cite déjà les noms de la Chapelle de Bragny, Berné, Lainé, Jalogny promus à un bel avenir. Et dans l'organisation du monastère clunisien, le garde du vin (custos vini) a autant d'importance que le celérier, le grènetier ou le jardinier. Et d'une manière plus générale, il n'est pas exagéré de dire que l'ordre de Cluny a joué pour le vignoble du Mâconnais, mutatis mutandis, le rôle qu'a tenu l'Ordre de Cîteaux pour le vignoble de la Côte bourguignonne. Au sud-ouest de Mâcon, le monastère clunisien de Saint-Martin des Vignes en conserve le souvenir et la toponymie (dom Charvin, Atlas des monastères de l'Ordre de Cluny au Moyen-Age, Paris, Ed. de Boccard, 1977).

ce qu'il est coutume d'appeler la "mosaique des comtés carolingiens", dont la plupart ont pu former les «pagi » ou " pays» constitutifs du duché de Bourgogne, le comté de Mâcon, correspondant au "pagus Matisconensis» a toujours bien été individualisé dès l'époque carolingienne. De plus, le morcellement physique du Mâconnais, entre val Clunisien et val de Saône, a favorisé l'émiettement de la propriété seigneuriale et paysanne. L'abbé Eugène Jarry (Provinces et pays de France, Tome III, Bourgogne, 1948) rappelle que « l'existence de paysans possesseurs (il s'agit surtout de vignerons dans les cas cités par A. Deléage) est certaine au moins dans le Mâconnais, du $\mathrm{X}^{\mathrm{e}}$ siècle et du début du XI ${ }^{e}$ siècle » (p. 189).

Le comté du Mâconnais conserva ainsi son identité au cours des siècles médiévaux, passant de la suzeraineté des marquis d'Auvergne à celle des ducs de Bourgogne. Pendant quelques décennies (1435-1477), le comté devint possession de ces derniers, avant d'être rattaché au royaume de France à la mort du Téméraire. Toutefois, la Saône demeure, ce qu'elle est encore aujourd'hui, une limite territoriale dans son rectiligne cours mâconnais entre « Royaume » à l'Ouest en rive droite, (aujourd'hui département de Saône-et-Loire) et « Empire » en rive gauche (aujourd'hui département de l'Ain).

11 Cependant, le vignoble, lui, dans les panneaux calcaires faillés, est entièrement localisé à l'ouest de la Saône, hormis le petit éclat de calcaire à l'est de Tournus (vins de Lacrost et Préty).

12 Ces quelques traits d'histoire devaient être rappelés car ils expliquent à la fois la spécificité historique et géographique du vignoble mâconnais, ses références et sa recherche d'identité entre les deux grands vignobles qui l'encadrent, bourguignon au nord et beaujolais au sud. 
13 De son rattachement au duché de Bourgogne, le Mâconnais a suivi, du moins pour ses vins blancs, la recherche de qualité par laquelle les grands ducs ont cherché à promouvoir les cépages nobles de pinot et au contraire à interdire les cépages de gamay. Il y a lieu de rappeler ici le célèbre édit de 1395 par lequel le duc Philippe le Hardi ordonne d'arracher de Bourgogne le «très mauvais et très déloyaux plant nommé gamay " ce plant donnant selon le même édit un vin "horrifique et puant». Dans les siècles qui suivirent, chaque fois qu'il s'agira de marquer leur différence par rapport au vignoble du Beaujolais entièrement tourné avec le gamay vers une production de quantité, les marchands mâconnais mettront en avant cette différence.

14 Ainsi, Roger Dion (Histoire de la vigne et du vin en France p. 377) citant les Archives départementales de Saône-et-Loire C.534, rappelle qu'à l'occasion d'un procès intenté à partir de 1616 par les échevins de Mâcon contre ceux de Villefranche-sur-Saône, les premiers ne manquèrent pas de souligner vivement que les seconds ne produisaient qu'à partir « de gamay... qui est une espèce de raisin l'usaige duquel est interdict en tout plain d'endroictz, mais spécialement au comté de Bourgogne, pour estre de sa nature grandement corrosif $»$.

15 Parce qu'ils étaient produits à partir de ce cépage de pinot blanc ou chardonnay (qui est d'ailleurs le nom d'un village du Mâconnais viticole... ), les vins blancs du Mâconnais, considérés comme "vins ordinaires de France » sans que ce mot ne revête le caractère plutôt peu qualifiant qu'il a de nos jours, étaient prisés du marché parisien où ils arrivaient après voyage terrestre par le Charolais, aux ports fluviaux de la Loire roannaise, d'où ils gagnaient Paris par le «canal de Montargis ». C'est alors que pour ces transports le vignoble du Mâconnais acquit sa particularité par rapport au vignoble de la Côte bourguignonne, en utilisant la «pièce mâconnaise » d'une contenance de 215 litres, différente de la «feuillette » bourguignonne d'une contenance de 228 litres. De même la « bouteille » mâconnaise fait 0,80 litre.

16 Avec ces contenances bien identifiées, les vins du Mâconnais circulent par les monts du haut-Charolais granitique descendant jusqu'à Roanne ou Pouilly-sous-Charlieu et sont ainsi exempts de droits "jusqu'au bureau de Nemours", comme le rappelle un marchand mâconnais en $1729 .$.

17 C'est que, vers le sud, les vins du Mâconnais se heurtaient à vive concurrence du vignoble du Beaujolais placé dans la mouvance et sous la protection de la ville de Lyon par de lourds péages dont devaient s'acquitter les marchands mâconnais dès leur arrivée au port de Villefranche-sur-Saône.

On peut dire sans erreur que c'est cette contrainte douanière qui, en fermant le vignoble mâconnais au marché lyonnais au profit du vignoble beaujolais, contraignit le premier à une recherche de qualité et l'obligea, avec celle-ci, à chercher les débouchés vers les pays du Nord et notamment Paris. Cependant qu'assuré du massif marché lyonnais, le vignoble du Beaujolais se contentait sur ses sols gréso-cristallins, avec le cépage de gamay, d'une production de quantité de vins agréables mais de faible garde...

19 Les archives sont remplies de ces querelles qui étaient loin d'être à sens unique... car le péage de Mâcon, dont on connaît l'existence depuis 812 jusqu'à la Révolution, était fort actif (A. Guerreau, la Saône axe de civilisation, pp. 365 à 383) et additionnait, à partir du $\mathrm{XVI}^{\mathrm{e}}$ siècle, le péage du roi, celui de l'évêque et celui des familles Chevrier et La Salle. 
20 De même, les édiles de Mâcon ne se privaient pas de taxer à leur tour « toute botte de vin de Beaujolais, même pour passer debout" (Mémoires des prévôts des marchands et échevins de Lyon et de ceux du Beaujolais, 1722, Arch. Nat. H 101).

21 Enfin, s'il est vrai que les vignerons du Mâconnais ont très tôt misé pour la production de leurs vins blancs sur le cépage pinot blanc chardonnay, ils ne se sont pas interdits, loin de là, malgré leurs protestations, d'utiliser, notamment pour produire des vins rouges, des cépages de gamay, mais il est vrai d'un " petit gamay » ou "gamay fin » venant bien sur le Mâconnais méridional cristallin qui fait transition avec le Beaujolais proche, de même nature géologique.

22 Cette dualité historique et géologique fait encore de nos jours la spécificité du Mâconnais, tirant vers le Beaujolais par ses rouges au sud, se rattachant à la Bourgogne de la Côte chalonnaise beaunoise et nuitonne par ses blancs, dès que l'on arrive sur les terroirs marno-calcaires et calcaires.

23 L'imbrication du socle cristallin et de sa couverture sédimentaire Secondaire dans un contexte très faillé est le trait physique dominant du Mâconnais qui explique aussi cette dualité.

\section{Fondements physiques de la viticulture mâconnaise}

Hormis les petites collines calcaires de rive gauche de la Saône à l'est de Tournus (petit vignoble de Lacrost-Préty déjà mentionné), la très grande majorité du Mâconnais viticole se situe en rive droite de cette rivière entre celle-ci et à l'Ouest le val de Grosne et de Guye, qui s'enfoncent dans les compartiments faillés de la bordure cristalline orientale du Massif Central à cet endroit.

C'est en effet par une vigoureuse tectonique en panneauxque le socle cristallin et sa couverture mésozoïque plongent vers le fossé bressan entre le horst du Mont SaintVincent, le Haut Charolais le Nord du Beaujolais et le val de Saône (fig. 1). 
Figure 1 : Lithologie du Mâconnais.

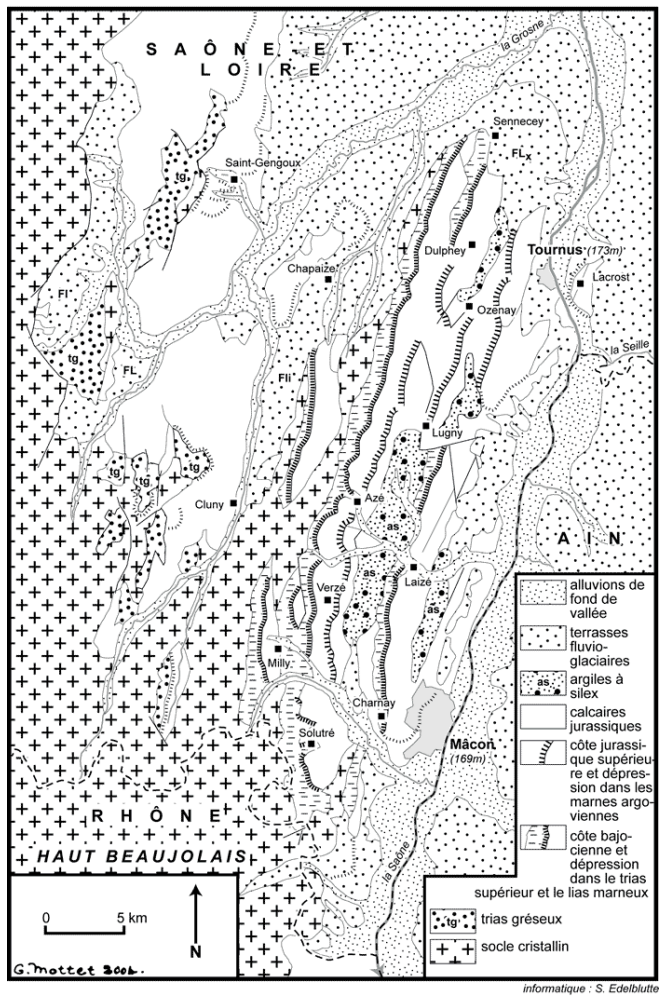

Avec un fort pendage vers l'est, les panneaux faillés constituent une alternance serrée de longs « chaînons » ou échines granitiques ou calcaires. Dans le second cas l'érosion différentielle a pu dégager et conserver de beaux éperons de calcaires durs au front élancé orienté vers l'ouest avec falaise rocheuse abrupte et revers structural en dalle plongeant vers l'est, soit en direction du front du panneau faillé le plus proche, soit sous le remplissage des terrains cénozoïques du fossé bressan.

27 Ainsi alternent sur une largeur n'excédant pas trente kilomètres, vallées, fronts abrupts de calcaires durs et leurs revers structuraux, échines granitiques, et à nouveau fronts et revers de panneaux calcaires faillés, entre chacun d'eux, de longs et étroits couloirs (fig. 1).

Cette tectonique vigoureuse et serrée est le propre du paysage morphologique mâconnais. La célèbre " roche de Solutré » en est le symbole (photo 1), rehaussée de sa richesse archéologique d'où elle tire un nom d'époque préhistorique, le "Solutréen ». $\mathrm{Au}$ pied de l'éperon célèbre comme sur son revers, les vignobles s'étendent sur les reliefs comparables, de Vergisson au Nord, de Pouilly, Fuissé, Vinzelles au Sud. 
Photo 1 : La roche de Solutré, symbole du Mâconnais viticole et préhistorique.

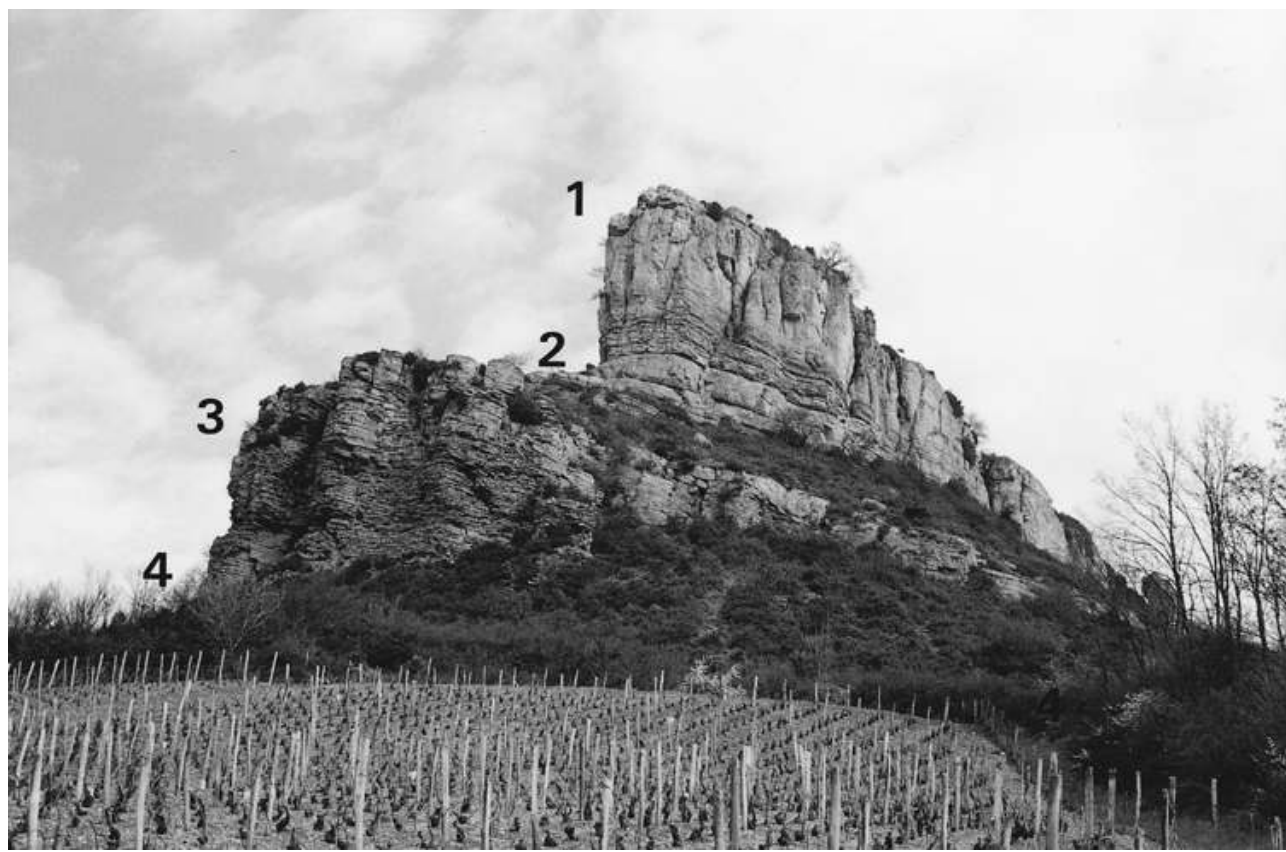

1, corniche de calcaire récifal, massif et résistant du Bajocien moyen ; 2 , replat dans les calcaires marneux (Bajocien inférieur) ; 3 , seconde corniche à Pecten Pumilus (Aalénien) ; 4, éboulis boisés cryoclastiques à vestiges préhistoriques ; à droite, le célèbre « Crot du Charnier »; 5 , vignoble d'appellation Mâcon-Solutré-Pouilly.

Photo Gérard Mottet.

29 À la faveur d'accidents transverses, cette tectonique orientée Nord $7^{\circ}$ à $10^{\circ} \mathrm{E}$ est aérée par plusieurs vallées réunissant chaque couloir. La plus marquée est celle de la " petite Grosne » débouchant dans le val de Saône au Sud de Mâcon. C'est par cette vallée que, par le col des Bois Clairs, le val de Saône peut communiquer avec le "val lamartinien » (Milly Lamartine, Pierreclos) puis avec le val clunysien.

C'est par cette échancrure également que, grâce à de nombreux ouvrages d'art passent la « route Centre Europe Atlantique » ou RCEA de Mâcon à Charolles et le TGV ParisLyon avec sa gare de Mâcon-Loché du nom d'un village viticole proche.

Cette complexité structurale sur une faible surface explique la grande diversité des sols, accentuée en plus par les héritages morpho et pédo-climatiques et les pénétrations en golfes entre les chaînons de la sédimentation marine ou lacustre cénozoïque du fossé bressan au rythme de la formation tectonique et de son remplissage alluvial.

D'une manière très générale, il ressort de cette diversité physique une hiérarchisation de l'aptitude des sols àla viticulture qui peut ainsi être exprimée, des meilleurs aux moins aptes :

1. Les meilleurs sols où se complaisent les cépages de pinot blanc chardonnay sont, comme dans la Côte bourguignonne, les calcosolscaillouteux formés sur les calcaires en plaquettes du Jurassique moyen (Bathonien) et des calcaires en plaquettes à alternance de niveaux marneux du Jurassique Supérieur (Kimméridgien). Le délitage en plaquettes favorise en effet la « pierrosité » des sols, propice à la vigne.

2. Cette pierrosité peut également exister sur les niveaux plus marneux ou marno-calcaires, lorsque la morphodynamique et la gélifraction quaternaires ont favorisé le transfert sur les versants des éléments pierreux des assises sus-jacentes plus rocheuses (marnes du Lias, 
marnes à Pholadomyes du Bathonien supérieur, marnes et marno-calcaires du Jurassique moyen et Supérieur (Callovien, Oxfordien).

3. Les conglomérats à éléments calcaires facilement altérables et ameublis en surface de SaintMaurice de Satonnay (formations m4c).

4. Les limons pliocènes, lorsqu'ils sont peu épais et bien exposés (Lugny) sont plantés en vignes.

5. Les formations superficielles (épandages caillouteux calcaires des terrasses les plus anciennes de la rive droite de la Saône) peuvent, selon la granulométrie et le PH, être également plantées en vignes (nord et sud de Mâcon).

6. Les sols sur socle (vieux schistes, séries volcanosédimentaires très fracturées, micro-granites roses très fissurés) du Mâconnais granitique, en général à altitude plus élevée annonçant le Haut-Charolais et Beaujolais cristallin, ne peuvent recevoir que les cépages de gamay qui, de ce fait, les excluent des appellations communales des pinots blancs. Ce sont plutôt les «beaujolais » du Mâconnais dans sa toponymie administrative départementale, appelés également «Mâcon rouge ". Il s'agit alors d'AOC régionales et non communales (Serrières sur le complexe des tufs et rhyolites houillers d'âge " probablement viséen » (notice carte géologique Mâcon, BRGM p. ll).

7. Les sols sur bas fonds alluviaux et humides des lits majeurs et d'inondation des vallées de la Grosne et de la Guye sont évidemment réservés aux pâturages ou forêts ripicoles et excluent la vigne de quelque appellation que ce soit.

8. Il en est de même des formations détritiques d'argiles à silex d'âge éocène (g3-m3) dites aussi formation de Verchiseuil. Cette formation peut aussi se présenter sous forme d'un conglomérat silicifié, dur. Le tout, abandonné à la forêt (bois de Naisse, de Verzé, de la Roche, du Chateau) exclue la vigne.

33 Cette diversité des potentialités pédologiques, conséquence du fractionnement morpho-structural, se combine aux multiples expositions, multipliant ainsi les potentialités viticoles, que le climat d'ensemble, à son tour, favorise.

Dans sa lyrique présentation de la Bourgogne (1936) Gaston Roupnel qualifie le Mâconnais de "Midi lumineux de la Bourgogne». Réflexion pertinente que confirment les données climatiques, les usages architecturaux (tuile ronde ou romaine, toits à faible pente) la biogéographie.

Allongées en rive droite $\mathrm{du}$ val de Saône, les collines calcaires du Mâconnais, participent des effets positifs, surtout pour la vigne, de la remontée thermique et d'ensoleillement du climat méditerranéen par le couloir rhodanien et le fossé bressan. Mais cette influence bénéfique est à nuancer. En effet si elle est réelle dans le Mâconnais méridional et en bordure du val de Saône elle s'atténue assez vite dans le Mâconnais central, le haut Mâconnais, le Mâconnais occidental et ses vallées en cul de sac où, au contraire, l'hiver, des coulées d'air froid venues des anticyclones continentaux du nord-nord-est de l'Europe peuvent venir s'installer durablement. Enfin par l'ouest arrivent évidemment les influences océaniques pluviogènes qui passant assez facilement entre le sud du Morvan et le nord-Charolais et viennent fortement arroser, en pluie comme en neige le horst cristallin du Mont-Saint-Vincent, le haut Mâconnais et son prolongement dans les collines calcaires par l'échine de granite du Mont-Saint-Romain (579 m), commencée au sud par le Signal de la Mère Brottier (758 m).

De telle sorte qu'à la diversité orographique correspond une réelle diversité climatique qui a conduit la commission des experts sur les AOC du Mâconnais par l'INAO à 
distinguer quatre types de milieux climatiques (Bouchard Ch., Chrétien J., Fermond C., Leneuf N., Mottet G., I.N.A.O. centre de Mâcon, rapport général sur l'AOC Mâcon, 2004, pp. 18-19).

\section{A. Le Haut-Mâconnais}

37 C'est par le soubassement cristallin soulevé et faillé que se fait le relèvement d'altitude qui prolonge en Mâconnais le Haut-Charollais et le Haut-Beaujolais voisins, de part et d'autre du val Larmatinien. Ces hauteurs comprises entre 600 et $758 \mathrm{~m}$ (Mère Brottier), ont des températures moyennes de janvier comprises entre $0,5^{\circ}$ et $0,9^{\circ} \mathrm{C}$ et de juillet entre $17^{\circ}$ et $17,9^{\circ} \mathrm{C}$ et des précipitations moyennes annuelles comprises entre 900 et $1300 \mathrm{~mm}$. La vigne est exclue de ces hauteurs propices à l'herbe, à l'élevage et à la forêt.

\section{B. Le Mâconnais central}

38 C'est l'ensemble des longues vallées encadrées de corniches calcaires qui s'alignent entre le val clunisien à l'ouest et les terrasses mâconnaises bordant la rive droite de la Saône de Tournus au nord à Mâcon au sud.

C'est là que s'alignent les vignobles en longues bandes parallèles entre chaque suite de corniches (photo 2) : alignement de Verzé, Igé, Azé, alignement de Lugny-Ozenay, ou de Péronne-Burgy-Chardonnay, par exemple.

Photo 2 : Vignoble du Mâconnais central (Azé), en enfilade entre les échines faillées.

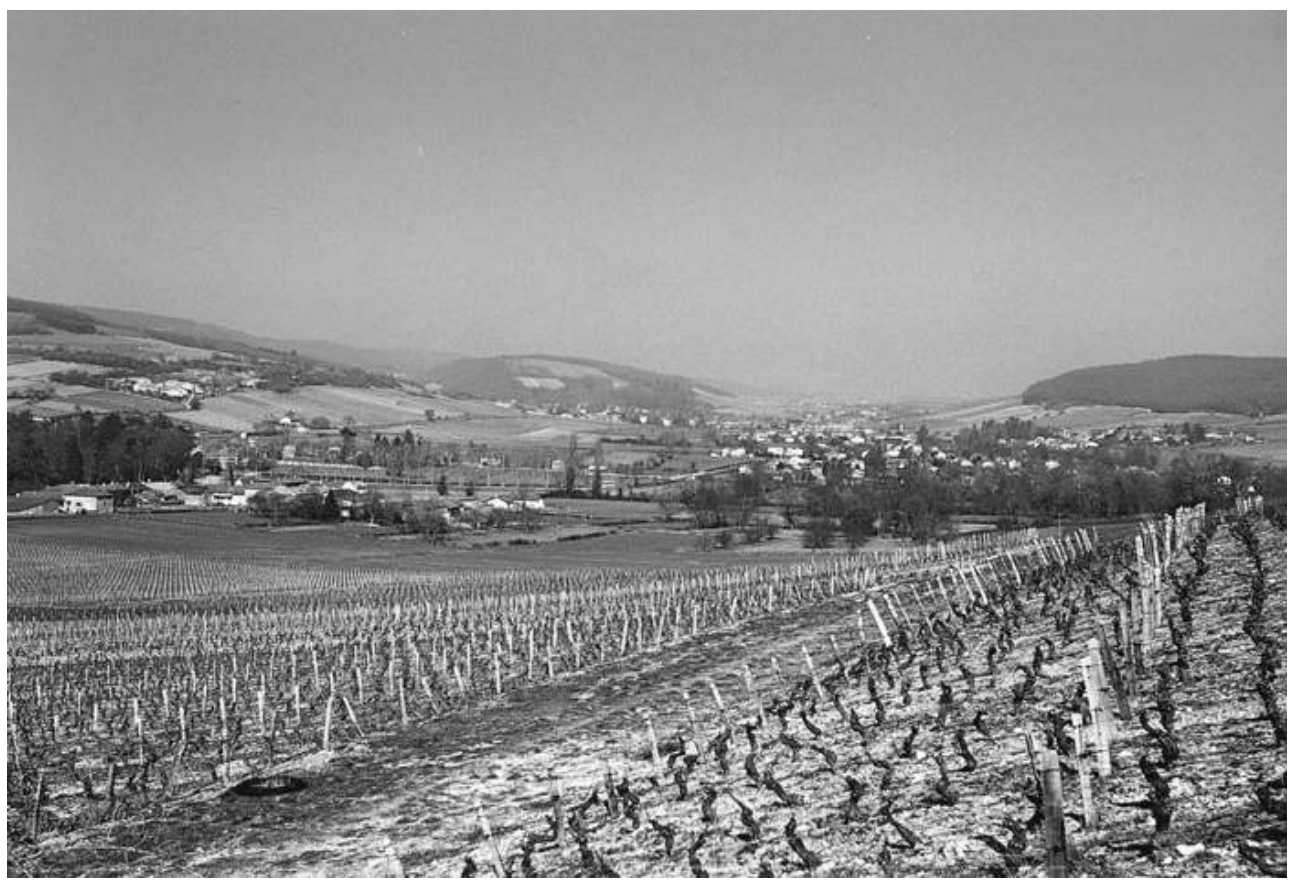

Photo Cédric Fermond, INAO, Mâcon.

Ces vallées allongées et leurs versants, comprises entre 150 et $300 \mathrm{~m}$ d'altitude, ont des températures moyennes de janvier comprises entre $1,5^{\circ}$ et $1,9^{\circ} \mathrm{C}$ et de juillet entre $19^{\circ}$ et $19,9^{\circ} \mathrm{C}$, et des précipitations moyennes comprises entre 700 et $900 \mathrm{~mm}$. Le froid 
hivernal d'origine continentale peut s'insinuer et stagner dans les vallées, cependant que les versants des coteaux mieux exposés sont plus ensoleillés et se réchauffent plus vite au printemps, échappant aux coups de gel de cette saison délicate entre toutes.

\section{Le Mâconnais « saônois »}

Entre l'ensemble précédent et le val de Saône s'étend une longue bande de terrasses et paliers faillés sur lesquels s'appuie au sud la majeure partie de l'agglomération mâconnaise et de la longue suite de villages qui la prolongent au nord-nord-est Sancé, Sennecé-lès-Mâcon, St Martin-Belleroche, Senozan, la Salle, le tout en un palier dominant la Saône d'une trentaine de mètres. Les vignobles longent la voie ferrée et l'autoroute. Cette longue marche procède du réchauffement estival du val de Saône et du moindre froid de l'hiver $\left(2^{\circ}\right.$ à $2,5^{\circ} \mathrm{C}$ de température moyenne de janvier), toutefois péjoré par les nappes de brouillard propres au val de Saône tout entier. La situation d'abri par rapport aux reliefs du Mâconnais central contribue à minorer les précipitations toujours inférieures à $850 \mathrm{~mm}$.

Le vignoble ne serait pas ici en mauvaise situation, mais sa gestion est rendue plus difficile par l'incertitude et les maladies liées aux épisodes de brouillard.

\section{Le Mâconnais méridional}

43 On peut ainsi qualifier une petite région naturelle au sud-sud-ouest de Mâcon, où la retombée des reliefs sur le val de Saône est élargie, aérée, échancrée par une tectonique plus complexe, quadrillant plusieurs directions, Nord $20^{\circ} \mathrm{E}$, et Nord $45^{\circ} \mathrm{W}$. En profite la petite Grosne qui ouvre ainsi le passage du val de Saône au val lamartinien et clunysien par le col des Bois Clairs et les ouvertures secondaires qui se greffent sur lui. C'est sur cette percée lumineuse que retombent les revers des panneaux de Vergisson, de Solutré, Davaillé, Pouilly, Fuissé, Vinzelles, Chaintré mais aussi au Nord du TGV La Roche-Vineuse, Prissé, Berzé-la-Ville. 
Photo 3 : Château et vignoble de Chasselas (sud Mâconnais), témoin du rôle de l'aristocratie et de la bourgeoisie dans l'histoire viticole de la région.

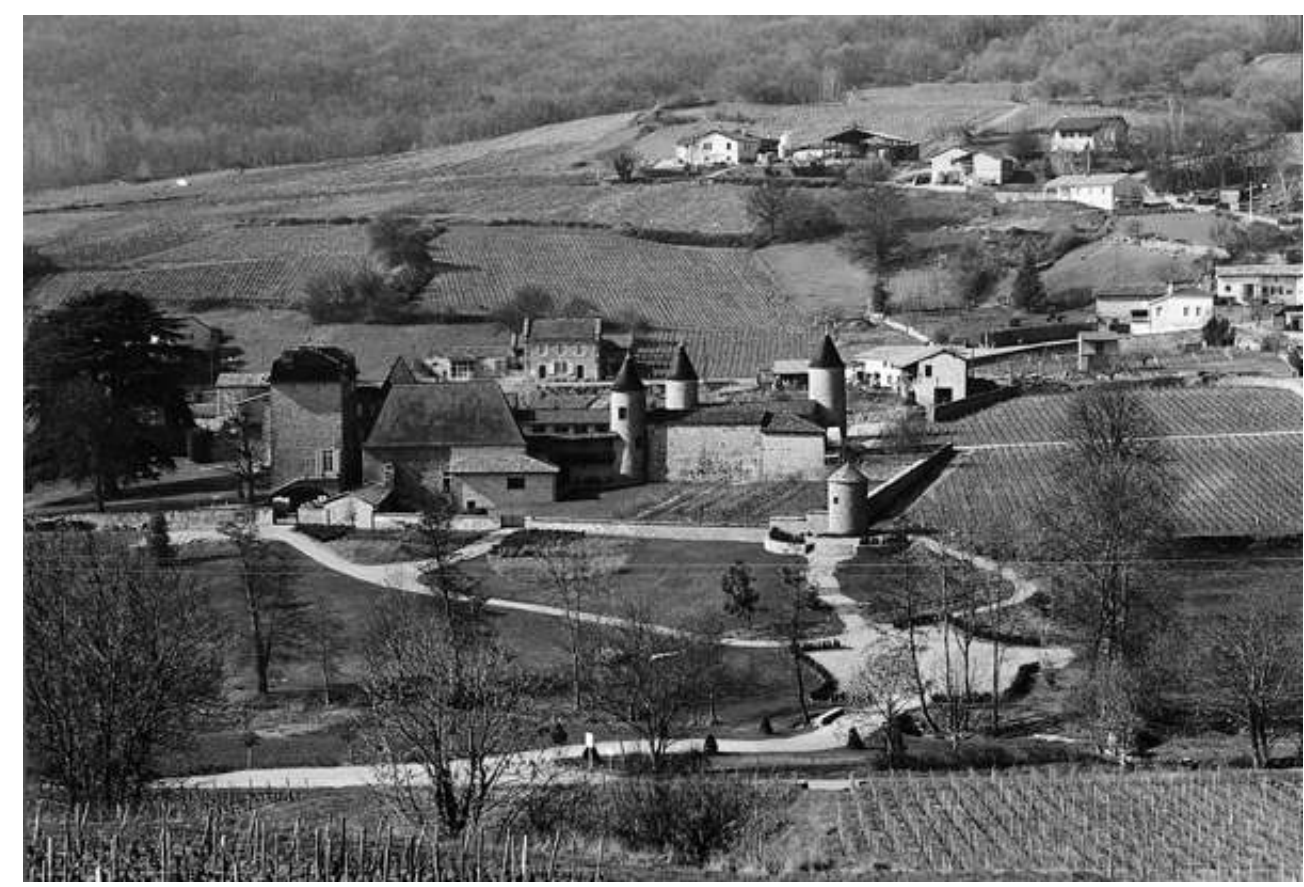

Photo Cédric Fermond, INAO, Mâcon.

Là sont les meilleures conditions climatiques et les blancs les plus prisés du Mâconnais, les meilleures appellations communales. Les remontées thermiques méditerranéennes d'été sont les plus sensibles avec un golfe de température moyenne de juillet net de $20^{\circ}$ à $25^{\circ} \mathrm{C}$, le plus élevé de tout le Mâconnais. C'est le Midi du Mâconnais, mais à la différence du Beaujolais proche, peut-être encore plus chaud l'été, sur de bons sols calcaires propices au pinot blanc. Solutré, Pouilly, Fuissé, Vinzelles forment le cœur historique et géographique des blancs fins du Mâconnais viticole, où se fait le mieux l'alliance du climat, du cépage et du sol. Une alliance déjà bourguignonne... mais cependant gardant son propre caractère, notamment par l'architecture de la maison vigneronne mâconnaise avec son toit à faible pente et à tuile ronde qui la distingue si fortement de celle de la côte beaunoise et nuitonne où règne la tuile plate ou vernissée (photo 3). Toutefois les belles caves creusées dans les solides assises calcaires jurassiques unissent l'ensemble...

\section{Les petites unités physiques du vignoble mâconnais}

En combinant le quadrillage climatique précédent et la fragmentation structurale et orographique du relief, on peut identifier un certain nombre de petites unités physiques ayant chacune leur caractère oro-climatique propre. Cette diversité n'est pas sans expliquer la multiplicité en Mâconnais des AOC communales et leur complexe imbrication avec les $\mathrm{AOC}$ régionales. 


\section{A. Le Haut Mâconnais cristallin}

Essentiellement recouvert de sombres forêts de résineux, le Haut Mâconnais cristallin est très peu viticole. On retrouve seulement la vigne dans les quelques dépressions calcaires (Blanot, Chissey, Château, Donzy) qui bordent les reliefs s'élevant entre 500 et $600 \mathrm{~m}$ d'altitude en moyenne. Ces reliefs SSW-NNE sont un important obstacle climatique qui concentre les perturbations et accentue les écoulements d'air froid sur les piedmonts. Cette zone est plus propice à la sylviculture et à l'élevage.

\section{B. La marge Beaujolaise}

47 Une amorce de dépression périphérique entre le socle granitique du Beaujolais et les panneaux calcaires basculés vers l'est, la marge beaujolaise, suit la vallée de l'Arlois qui vient buter en rive droite contre le puissant socle cristallin qu'elle érode en de larges mamelons bombés, s'enfonce dans les tendres marnes du Trias et du Lias et découpe les calcaires du Jurassique moyen en de longs versants rocailleux.

La vigne occupe l'intégralité des versants de la vallée et ne laisse place aux prairies que dans les fonds du vallon et sur les versants les plus mal exposés (fig. 2).

Figure 2 : Les vignobles du Mâconnais.

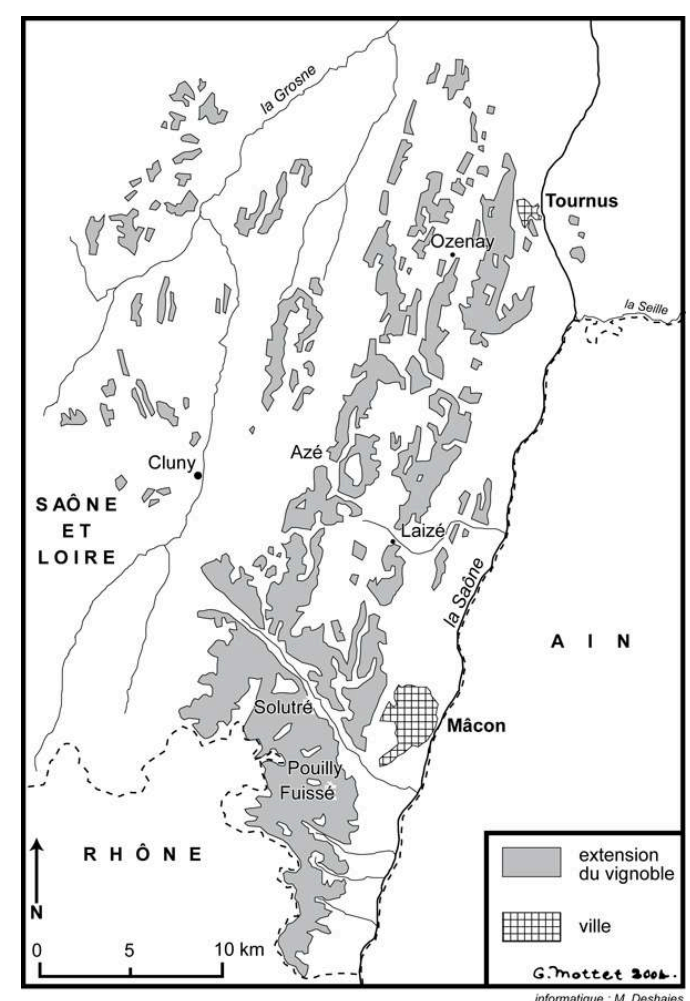




\section{Le haut val Lamartinien}

Blotti contre les monts granitiques et ouvert vers l'est par le cours de la petite Grosne et du Fil, le vignoble du haut val lamartinien est partagé entre l'acidité des granites, la lourdeur des marnes et la dureté des calcaires.

Chaud le jour et frais la nuit, la proximité des reliefs vient rajouter de la pente à ce vignoble déjà plus dispersé que dans le sud, et qui fit le bonheur et fut la source d'inspiration du célèbre poète Lamartine et de sa famille. Nombreux sont les travaux sur la viticulture publiés en particulier par François-Louis de Lamartine dans les Annales de l'Académie de Mâcon en 1806 et 1821.

\section{Les collines du Clunisois}

51 Les douces collines calcaires et marneuses du Clunisois sont tournées vers l'élevage. Même si quelques parcelles de vignes persistent sur les flancs ouest de courts côteaux des marnes liaso-triasiques et même si l'habitat traditionnel vigneron et les petites parcelles entourées de murets rappellent que la vigne était très présente dans le Clunisois (en liaison avec l'Ordre de Cluny), la viticulture est aujourd'hui une activité marginale.

\section{E. Les collines du val de Grosne}

Entourées par le remplissage alluvionnaire de la vallée de la Grosne et du Grison, quelques courtes collines calcaires émergent de la plaine et offrent de petits côteaux calcaires partiellement plantés en vigne, ouverts vers le sud et l'est. Sous influence du climat saônois, ce val est partagé entre les prairies bovines, les grandes cultures, l'exploitation forestière, l'élevage caprin et la viticulture.

\section{F. La côte sud-Chalonnaise}

Sur ce chaînon jurassique, quelques parcelles de vignes dispersées occupent en de rares endroits des côteaux calcaires bien exposés. Il est bordé de prairies consacrées au bœuf charolais et voit la grande culture remonter jusque vers ses ceps.

Le revers ouest est quasi intégralement une zone d'élevage. La vigne ne daigne pas traverser la vallée de la Guye et s'aventurer sur les terrains granitiques, plus froids et plus humides, du nord-Charolais et du horst élevé du Mont-Saint Vincent (603 m).

\section{G. Le palier oriental mâconnais pré-saônois}

Appuyé contre les derniers chaînons calcaires du Mâconnais central et regardant vers la plaine de la Saône, le palier oriental est déjà un avant-goût de la Bresse avec ses dépôts anciens de la Saône qui reposent en faible épaisseur sur un dernier gradin calcaire qui tarde à s'enfoncer dans le fossé Bressan. La vigne est marginale sur cette bordure plane. Les plateaux sont bien plus souvent voués à la grande culture, aux prairies et aux habitations de la banlieue de Mâcon qu'aux ceps de vigne et seuls quelques rares et courts talus sont parfois occupés par celle-ci. 


\section{H. Le Mâconnais central}

56 C'est un système de sillons SSW-NNE, découpés d'ouest en est par un réseau hydrographique très ramifié qui, au bénéfice de trois ou quatre cluses, traverse les panneaux calcaires, avant de rejoindre le cours de la Saône à l'est (fig.1).

La vigne, généralement regroupée sur les grands côteaux de calcaires et de marnes du Jurassique moyen et supérieur, se réserve très souvent les meilleures expositions (fig. 2). On la retrouve plus dispersée, sur les granites et les marnes du Lias et du Trias, le long de revers orientés vers l'ouest. Elle gagne ponctuellement les quelques côteaux armés par les conglomérats calcaires du Tertiaire ainsi que de rares plateaux limoneux du Pliocène. Le tiers inférieur des côteaux calcaires largement planté en vigne est très souvent recouvert d'épandages caillouteux quaternaires.

La vigne dans le Mâconnais central recouvre une très grande diversité de matériaux géologiques sous des expositions et des formes de reliefs très changeantes. Cette variabilité de situations est à l'origine de la diversité des vins du Mâconnais central. Une diversité qui se manifeste également dans le paysage agraire : le Mâconnais central est un système de polyculture vigne/élevage/grande culture même si, depuis les 20 dernières années, les fermes ont fortement tendance à se spécialiser. Ainsi, un même fermier peut fort bien conduire un élevage et une production de fromages de chèvres (pour lequel il sollicite d'ailleurs une AOC en cours d'obtention) et une production de vin blanc bénéficiant d'une $\mathrm{AOC}$ communale. La dégustation devient alors harmonieuse et complète...

\section{Le sud-Mâconnais}

59 Une région ouverte vers l'est et les voies de communication, où les cumuls de températures sont les plus élevés du Mâconnais et les précipitations les plus faibles, au relief disséqué par les ruisseaux qui ouvrent de longs côteaux rocailleux vers l'est et le sud. La vigne domine le paysage agricole (photo 3). Elle s'étend sur l'ensemble des collines du sud-Mâconnais et ne cède la place aux prairies ou aux bois seulement lorsque le relief et trop accidenté, les sols trop superficiels ou trop humides, ou les expositions trop au nord. Le vignoble du sud-Mâconnais présente tous les atouts d'une terre viticole de qualité.

60 Là, sont les AOC communales actuellement les plus connues de l'extérieur, PôuillyFuissé, Pouilly-Vinzelles, Saint-Vérand, Pouilly-Loché.

61 La logique climatique est donc bien confirmée.

\section{Usages, structures et AOC du Mâconnais}

62 Ainsi donc, de toutes ces petites unités physiques, ce sont les deux dernières qui, favorisées par les expositions, le sol calcaire et le climat sont les plus viticoles: en Mâconnais central la vigne couvre entre 10 à 50 \% de la SAU, dans le sud Mâconnais elle dépasse le seuil des $50 \%$.

63 Ailleurs, elle est plus dispersée dans l'association culture-bocage d'élevage de la race charolaise et élevage caprin, survivance de la polyculture d'antan qui est loin d'avoir 
dit son dernier mot en cette période d'incertitude de réformes successives de la PAC et de doute alimentaire. AOC Beaujolais, rouge avec gamay, voire «Beaujolais blanc» avec chardonnay (La Chapelle de Guinchay)... On voit bien à ce sujet combien il est important que la géographie soit présente pour rappeler l'impérative rigueur des milieux physiques dans ses rapports avec les « produits de terroirs »...

71 La production de vins en Mâconnais oscille autour de $300000 \mathrm{hl}$ par an dont $77 \%$ de blancs et $23 \%$ de rouges. $80 \%$ de cette production sont en AOC «Mâcon » et $20 \%$ en AOC régionales de Bourgogne. Ce système de repli des vins du Mâconnais en appellation régionale provoque de ce fait, au gré des années et des fluctuations du marché du vin et hélas des méventes (années 2003-2004), de fortes instabilités des volumes négociables. La commission des experts de l'INAO a beaucoup insisté sur ce point (rapport, opus cité, p. 32).

72 Dans tout le Mâconnais la vigne est palissée, pour les cépages rouges comme pour les blancs. Les différences portent sur les modes de taille et les densités de plantation.

73 Ainsi le tiers nord se présente comme un vignoble à la densité de plantation élevée (8000 à 10000 pieds à l'ha) avec la taille guyot prédominante. 
74 Les deux tiers sud ont une densité plus faible (moins de 8000 pieds à l'ha) et la vigne est souvent taillée en arcures (photo 4), notamment dans le Mâconnais central. La vendange mécanisée est prédominante.

Photo 4 : Vigne conduite en taille de « double arcure " propre au Mâconnais central et méridional (moins de 8000 pieds à l'hectare).

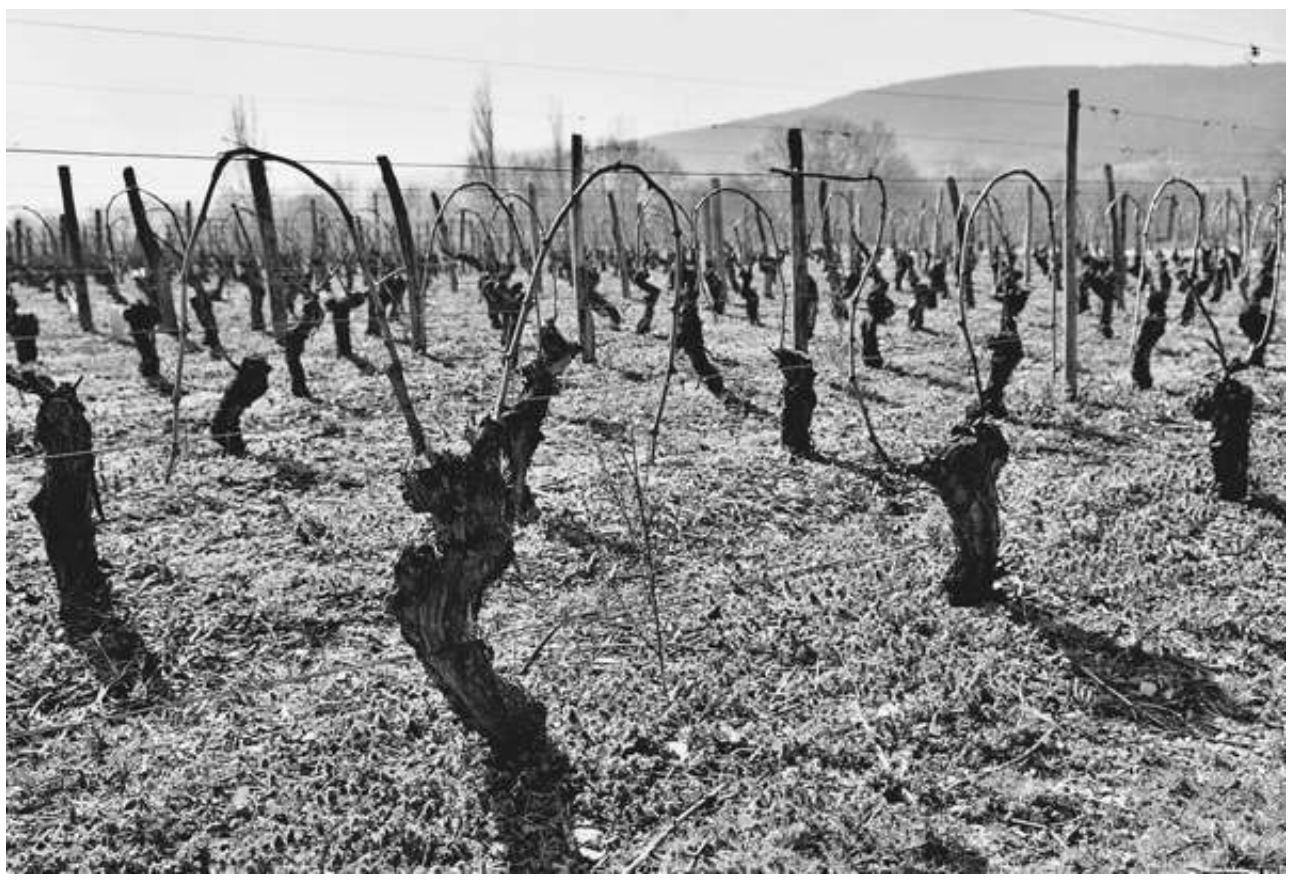

Photo Cédric Fermond, INAO, Mâcon.

Le système coopératif l'emporte. La moitié des vignerons sont coopérateurs d'une des 13 caves coopératives (environ 1500 coopérateurs).

76 Les vignerons du Mâconnais vinifient de façon très traditionnelle. Les raisins sont foulés ou laissés entiers comme en Beaujolais, les macérations sont courtes, les vins du Mâconnais se boivent jeunes. L'élevage en fûts est marginal, sauf pour les cuvées les plus soignées du Mâconnais méridional qui sont passées en fûts.

77 Le mode de faire valoir indirect est encore dominant: $44 \%$ des exploitants sont propriétaires, $24 \%$ sont des fermiers et $32 \%$ sont en métayage. La bourgeoisie mâconnaise reste encore très attachée à la propriété viticole et de ce fait, l'émancipation foncière est encore partielle.

Ce trait sociétal essentiel rattache le Mâconnais viticole au Charolais bocager proche, traduisant ainsi l'origine de ce vignoble lié au départ à la polyculture d'élevage, dont il fut longtemps un complément avant de s'en affranchir partiellement, notamment après la crise du phylloxéra.

\section{Conclusion}

79 Rien n'est donc simple dans ce vignoble à l'histoire, aux usages et aux structures aussi complexes que son cadre physique très fragmenté. Rien à voir avec la continuité géographique de la "Côte bourguignonne » Côte d'Orienne, celle du piedmont du 
Beaujolais, celle de la percée cataclinale du Chablisien au Nord, ou celle des collines sous-vosgiennes du vignoble alsacien. l'ensemble des AOC communales des blancs du Mâconnais, la production de vins rouges en "petit gamay », celle de Beaujolais rouge en Saône-et-Loire et maintenant blanc, risque de brouiller l'image d'une production qui, pourtant, accède dans l'ensemble à une réelle qualité.

\section{BIBLIOGRAPHIE}

BRÉjouX P.(1978). - Les vins de Bourgogne. Revue des vins de France, 275 p.

BRGM (1969-72-77). - Feuilles et notices cartes géologiques 150 000, Mâcon, Tournus, Cluny.

CHAMPIER L. (1959). - - Les fondements humains du vignoble mâconnais, Annales Acad. de Mâcon, tome XLIV, p. 65.

Dion R. (1977). - - Histoire de la vigne et du vin en France, Flammarion, Paris

JeAnton G. (1908). - La Bourgogne à Paris au Moyen-Age. Les vins du Mâconnais et la " rêve » mâconnaise à Paris au XIV ${ }^{\mathrm{e}}$ et XV $\mathrm{XV}^{\mathrm{e}}$ siècles (impôts) ? Ann. Acad. Mâcon, Tome XIII, p. 41

JouRnauX A. (1956). - Les plaines de la Saône et leurs bordures montagneuses. Thèse d'État, Caen.

LAMARTine F.L. (1806). - Des moyens d'améliorer le sort des vignerons en général et en particulier de ceux du Mâconnais. An. Acad. Mâcon 
MотTET G. (1999). - Géographie physique de la France, pp. 169 à 172, Charolais, Clunysois et Mâconnais, PUF, $3^{\mathrm{e}}$ éd., 768 p.

POMEROL C. et al. (1984). - Terroirs et vins de France, pp. 77-81, BRGM, 343 p.

RAT P. et al. (1972). - Bourgogne Morvan pp. 87 à 97, Monts du Mâconnais, coll. guides géol. régionaux, Masson, $174 \mathrm{p}$.

WILSON J.E. (1998). - Terroir, the role of geology, climate and culture in the making of French vines, Mâconnais, pp. 153-156, University of California Press, Berkeley, 336 p.

\section{RÉSUMÉS}

Entre la Côte bourguignonne au nord et le Beaujolais auSud, le vignoble du Mâconnais tire son originalité du relief très fracturé par la tectonique qui le compose. 43 appellations communales AOC Mâcon-villages en vins blancs (cépage chardonnay) sont la conséquence de cet émiettement, avec en plus connus Pouilly-Fuissé, Solutré, Saint-Vérand. Il a donc été nécessaire de proposer des regroupements dans les appellations.

Between Burgundy's coast in the North and Beaujolais up South the Mâconnais's vineyard stands out because it is the result of "eastward-tilted fault blocks". 43 villages are allowed to hyphenate their name with Mâcon itself in "Villages appellations", specially for white wines (chardonnay), the most famous being Pouilly-Fuissé, Solutré, Saint-Vérand. A new organisation of those appellations is required.

Zwischen der burgundischen Stufe im Norden und dem Beaujolais im Süden ist der Weinbau im Mâconnais eigenständig wegen seines durch die Tektonik stark zersplitterten Reliefs. 43 Gemeindeherkunftsbezeichnungen Mâcon-villages aus Weißweinen sind die Folge dieser Zersplitterung; unter den bekanntesten, sind Pouilly-Fuissé, Solutré, Saint-Vérand. Infolgedessen war es notwendig, Zusammenlegungen der Herkunftsbezeichnungen vorzuschlagen.

\section{INDEX}

Mots-clés : appellation village, chardonnay, Mâconnais, Pouilly-Fuissé, Saint-Vérand, Solutré, vin blanc

Schlüsselwörter : Chardonnay, Dörfer mit Herkunftsbezeichnungen, Mâconnais, Pouilly-Fuissé, Saint Vérand, Solutré, Weissweine, z.B.

Keywords : chardonnay, Mâconnais, Pouilly-fuissé, Saint-Vérand, Solutré, village appellation, white wine

\section{AUTEUR}

\section{GÉRARD MOTTET}

Professeur émérite des Universités, Vice-Président du Conseil Économique et Social de Bourgogne, expert INAO, 13 Grande Rue, 89113 Neuilly, France 